\title{
Giant Intrathoracic Meningocele and Breast Cancer in a Neurofibromatosis Type I Patient
}

\author{
Hridayesh Pratap Malla, M.D., ${ }^{1}$ Bong Jin Park, M.D., Ph.D., ${ }^{1}$ Jun Seok Koh, M.D., Ph.D., ${ }^{2}$ Dae Jean Jo, M.D., Ph.D. ${ }^{2}$ \\ Department of Medicine, ${ }^{1}$ Graduate School, Kyung Hee University, Seoul, Korea \\ Department of Neurosurgery, ${ }^{2}$ Kyung Hee University Hospital at Gangdong, Seoul, Korea
}

Intrathoracic meningoceles are relatively rare entities found in patients with neurofibromatosis type I (NF1). Given that both the BRCA1 and NF-1 genes are located on the same long arm of chromosome 17, one would expect concurrence of neurofibromatosis and breast cancer. However, incidence of such co-disorders is very rare in the literature. Here, the authors report a case of a 50-year-old female patient with NF-1 and concurrent cancer of the left breast, who had a huge bilobulated intrathoracic meningocele with thoracic dystrophic scoliosis, treated surgically via a posterioronly approach for the meningocele and spinal deformity in the same setting.

Key Words : Neurofibromatosis · Meningocele $\cdot$ Breast cancer.

\section{INTRODUCTION}

A spinal meningocele is a protrusion or herniation of the meninges through an intervertebral foramen or vertebral defect. It can be acquired following trauma or surgical procedures, including laminectomies, or can be of embryological origin. Meningoceles are rare entities usually found in lumbosacral regions. Intrathoracic meningoceles are even rarer, commonly associated with neurofibromatosis type I (NF-1). NF-1, also known as von Recklinghausen's disease, is an autosomal-dominant disease caused by genetic mutations of the NF-1 gene located on chromosome 17q11.2. Similarly, mutations of the BRCA1 gene on the long arm of chromosome 17 predispose a patient to breast cancer ${ }^{3)}$. Hence, there is a strong possibility of coexistence of breast cancer in patients with NF-1. However, such concurrence is scarcely reported in the literature, necessitating wider and more detailed investigations. Spinal deformities are common in patients with spinal meningocele. In fact, kyphoscoliosis is frequently found associated with posterior intrathoracic meningocele ${ }^{4}$. These patients frequently present with complaints related to compression of nerves or the spinal cord or due to respiratory symptoms resulting from an intrathoracic mass. Such patients need to be treated through surgical removal of the large mass and correction of the spinal deformities.
Here, we report a rare case of a 50-year-old female patient with a large bilobulated intrathoracic meningocele associated with NF-1 concurrent with invasive ductal carcinoma of the left breast.

\section{CASE REPORT}

A 50-year-old female complaining of dyspnea and bilateral upper limb numbness progressively worsening for the previous month was referred to the department of neurosurgery for further evaluation. She had been diagnosed with NF-1 about 20 years prior and had been recently diagnosed with left-sided breast cancer detected on Positron Emission Tomography (PET) scan. Histological investigations of the breast mass revealed the cancer to be invasive ductal carcinoma. There was no history of infection, trauma, or spinal surgery. On physical examination, diminished breath sounds were noted on the right. Also, there were widespread café-au-lait macules along with subcutaneous nodules and prominent bilateral freckling in the axillae (Fig. 1A, B). On neurological examination, no focal deficits were appreciated except for the right upper extremity weakness, grade III/V. Chest radiography showed a large mass-like lesion on the right lung associated with significant scoliosis (Fig. 2A). Computed tomography and magnetic resonance imaging of the lungs revealed a

- Received : December 31, 2014 • Revised : July 9, 2015 •Accepted : October 5, 2015

- Address for reprints : Dae Jean Jo, M.D., Ph.D.

Department of Neurosurgery, Kyung Hee University Hospital at Gangdong, 892 Dongnam-ro, Gangdong-gu, Seoul 05278, Korea

Tel : +82-2-440-6147, Fax : +82-2-440-7494, E-mail : apuzzo@hanmail.net

- This is an Open Access article distributed under the terms of the Creative Commons Attribution Non-Commercial License (http://creativecommons.org/licenses/by-nc/3.0) which permits unrestricted non-commercial use, distribution, and reproduction in any medium, provided the original work is properly cited. 

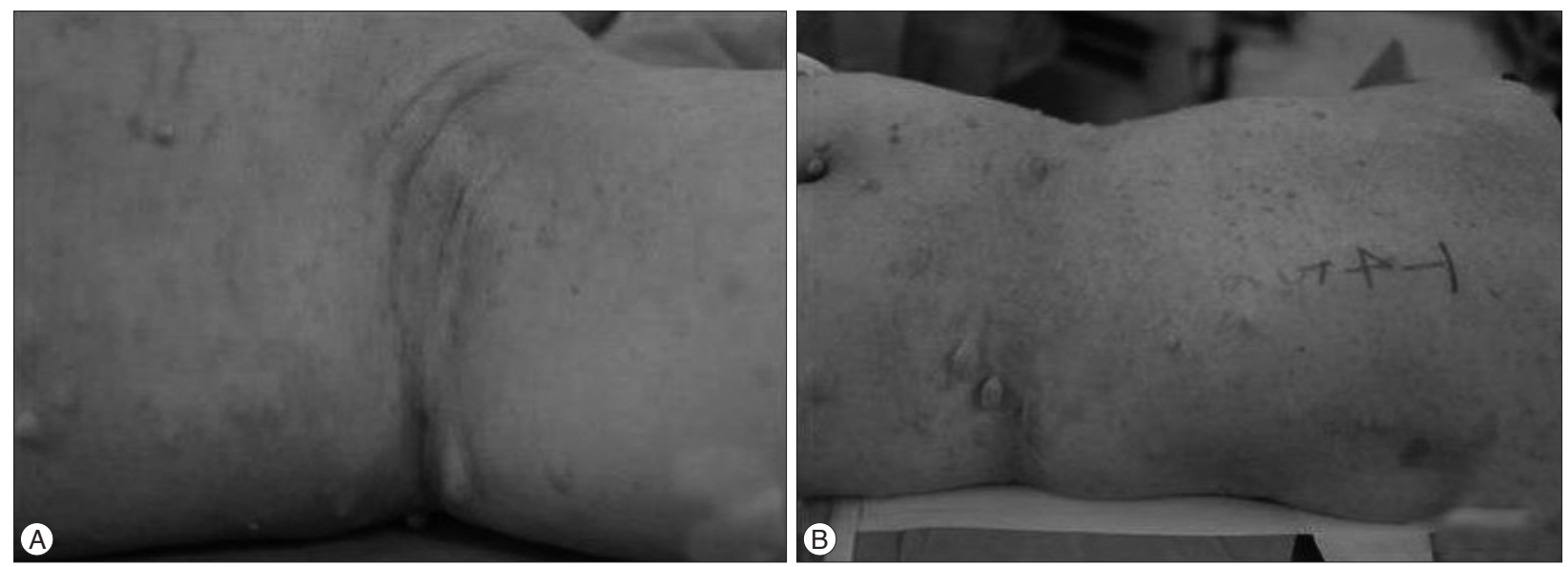

Fig. 1. (A) Photographs of the patient showing axillary freckling and (B) multiple subcutaneous nodules distributed all over the body.
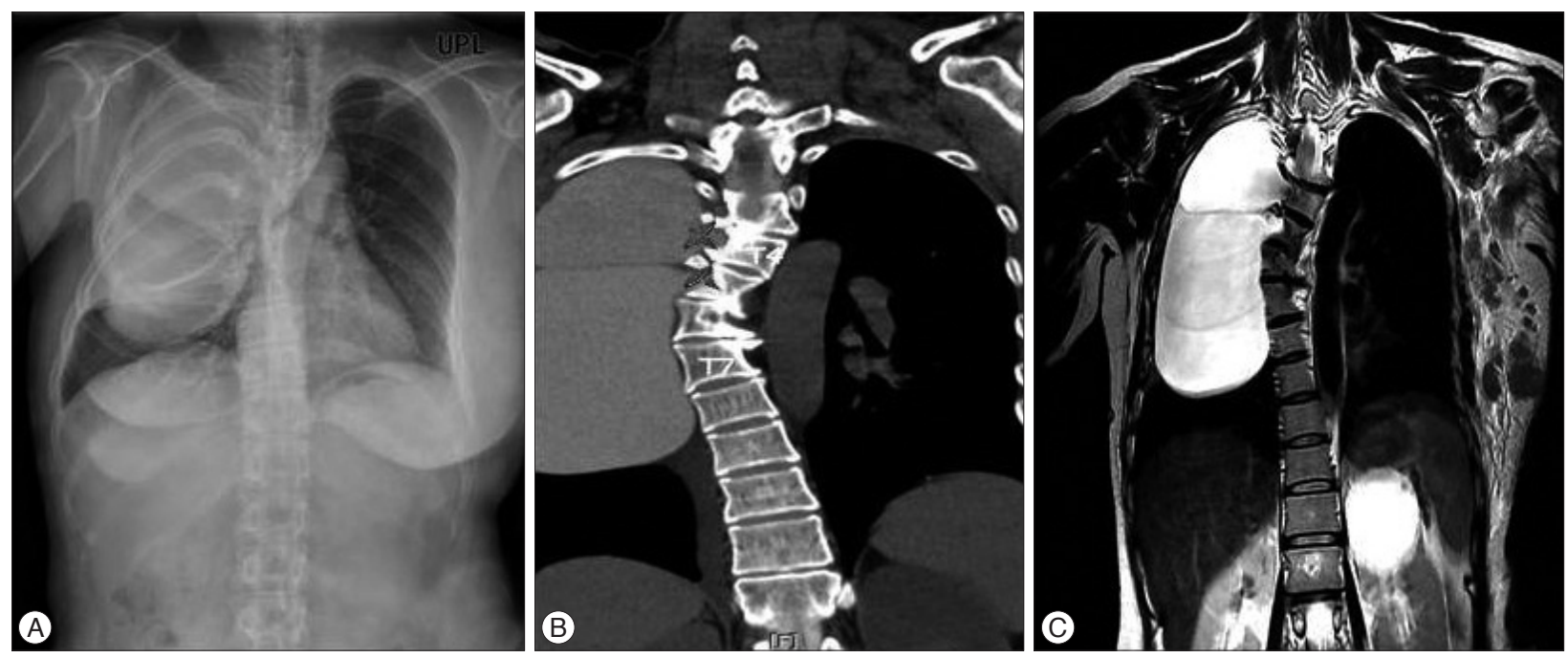

Fig. 2. A : Preoperative postero-anterior chest $X$-ray of the patient showing a hyperdense lesion in the right lung field with significant scoliosis of the thoracic spine. B : Preoperative myelo-CT scan showing a bilobulated, hyperdense cystic mass extending from the third to the eighth thoracic vertebral level, originating from the intervertebral foramen of the right fourth and fifth thoracic vertebrae (star-shaped marks). C : Preoperative T2W1 coronal MRI of the thoracic spine showing the hyperintense cystic mass on right side of the chest with scoliotic deformity.

thin-walled, bilobulated, homogenous cystic mass extending from the second to eighth thoracic segments (Fig. 2B, C). The right neural foramens of the fourth and fifth thoracic vertebrae were dilated, likely due to protrusion of the meninges via these foramens. There was also significant spinal cord compression at the fourth and fifth thoracic levels. A postero-laterally oriented intrathoracic meningocele was thus diagnosed. Scoliotic angle was calculated to be approximately 42 degrees by Cobb's method. Upper extremity weakness could not be explained by the thoracic mass, and so electromyography analysis was performed, revealing left brachial plexus injury. This injury was believed to be due to the left axillary mass, specifically lymph node metastasis from the breast cancer, as shown in a PET scan (Fig. 3).

Due to the large size of the mass and severe dyspnea, surgical removal and deformity correction surgeries were planned. Pedicle screws were first inserted on the left concave side of third to ninth thoracic vertebrae. The scoliosis curve was then corrected with a rod via the derotational technique, and pedicle screws were inserted on the right convex side at the third, seventh, eighth, and ninth thoracic vertebra (Fig. 4). The cystic mass was removed after performing total laminectomies and resection of the right T4 and T5 transverse processes, including removal of the proximal heads of the right fourth and fifth ribs (Fig. 5). As shown in the Fig. 5B, two openings of the meningocele into the spinal cord through the intervertebral foramen were found, and cerebrospinal fluid (CSF) was drained from the meningocele. Since the origin of the mass was the ventro-lateral portion of the spinal cord, direct closure of the dura could not be performed easily. Therefore, closure was achieved using a subcutaneous fat graft. The surgery proceeded without any adverse events.

Chemotherapy was started two weeks after surgery, and the patient was followed for about 30 months post-operatively. However, the follow-up X-ray images (Fig. 6) 15 months after the initial surgery showed recurrence of the meningocele in the upper 
lobe of the right lung and satisfactory correction state of kyphoscoliosis. Since there were no respiratory symptoms, conservative management was planned, and the patient was closely followed. After 30 months of close follow-up, the patient expired due to multiple metastases and systemic illness.

\section{DISCUSSION}

The association between intrathoracic meningoceles and NF1 has been explained in a literature review by Andrade et al. ${ }^{5}$, which stated that about $69 \%$ of intrathoracic meningoceles occurred in association with NF1, while only $22.4 \%$ occurred as isolated pathologies. Various theories have been postulated regarding the etiology of intrathoracic meningoceles in the presence of NF1. Laws et al. ${ }^{7}$ found in their radiological survey that about $22 \%$ of patients with NF1 had enlarged intervertebral foramen, and $18 \%$ had erosion of the pedicles. In such regions, the

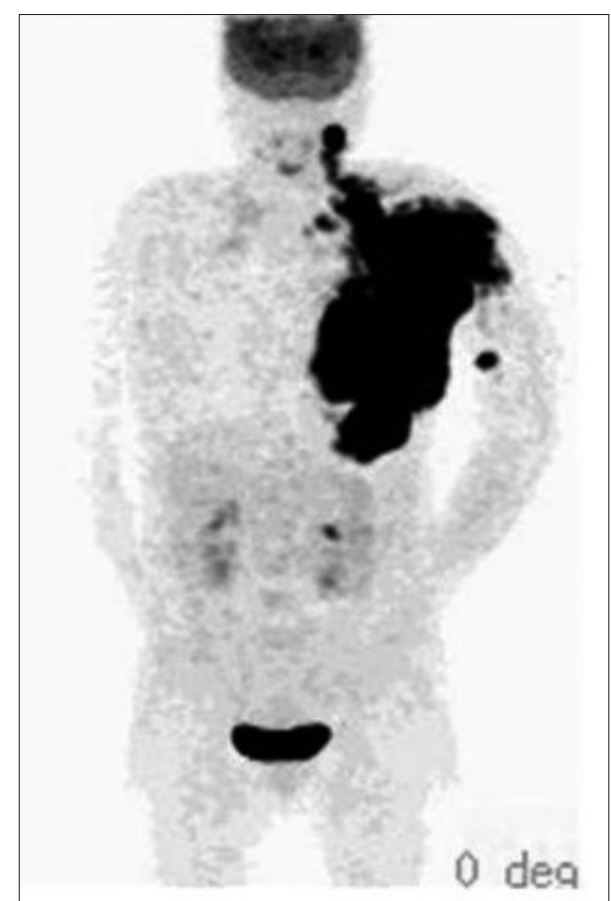

Fig. 3. Post-operative PET scan of the patient showing increased uptake in the region of the left breast suspicious for breast carcinoma. subarachnoid space was also found to be sufficiently expanded to pass through the intervertebral foramina in the form of saccular pouches. However, the embryonic origin of these meningoceles is still unknown.

NF1 is an autosomal-dominant disorder with a prevalence of 1 in $3000^{6,15)}$. It is also associated with various other malignancies such as peripheral nerve sheath tumors, optic gliomas, and leukemias. The diagnostic features of NF1 include the presence of café-au-lait macules, subcutaneous nodules, freckling in the axillary or inguinal regions, optic gliomas, and Lisch nodules. In our case, café-au-lait macules, axillary freckling, and subcutaneous nodules were present. In a case series, Nanson et al. ${ }^{10)}$ reported that about $70 \%$ of intrathoracic meningoceles were associated with NF1 and scoliosis, referring to the cluster of these disorders as a three-fold syndrome. In our case as well, the patient was known to have NF1 and presented with a giant intrathoracic meningocele and significant scoliosis. Controversies regarding the treatment of intrathoracic meningocele are prevalent among scholars. Miles et al. ${ }^{8)}$ advocated conservative treatment

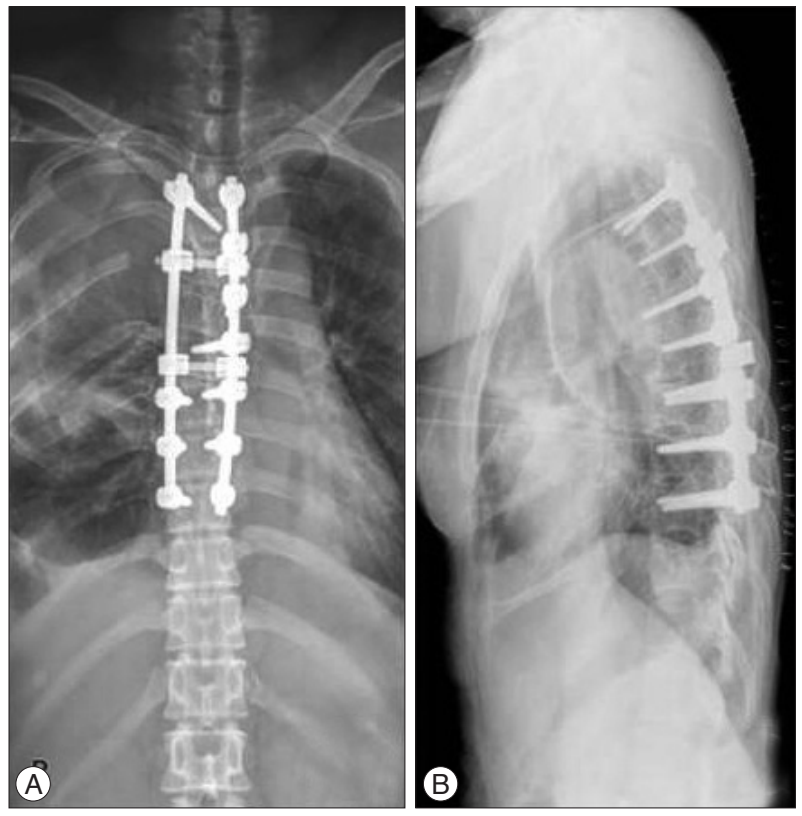

Fig. 4. Postoperative antero-posterior (A) and lateral (B) chest radiographs showing complete removal of the intrathoracic meningocele with significant correction of the scoliotic angles with screws and rods in situ.
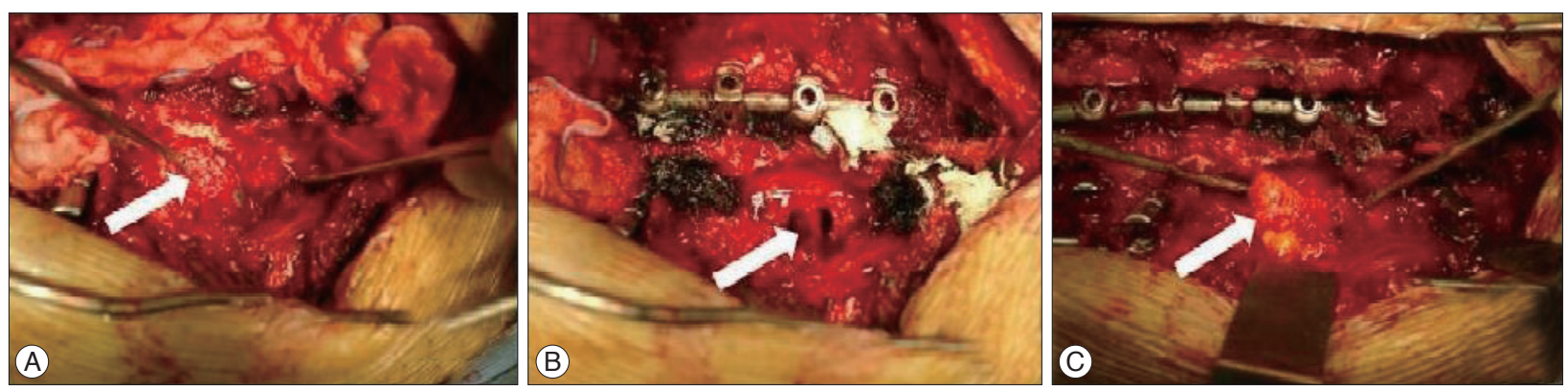

Fig. 5. A : Intraoperative photograph showing meningocele (arrow). B : Defect formed after excision of the cyst (arrow). C : Obliteration of the defect with the subcutaneous fat graft (arrow). 


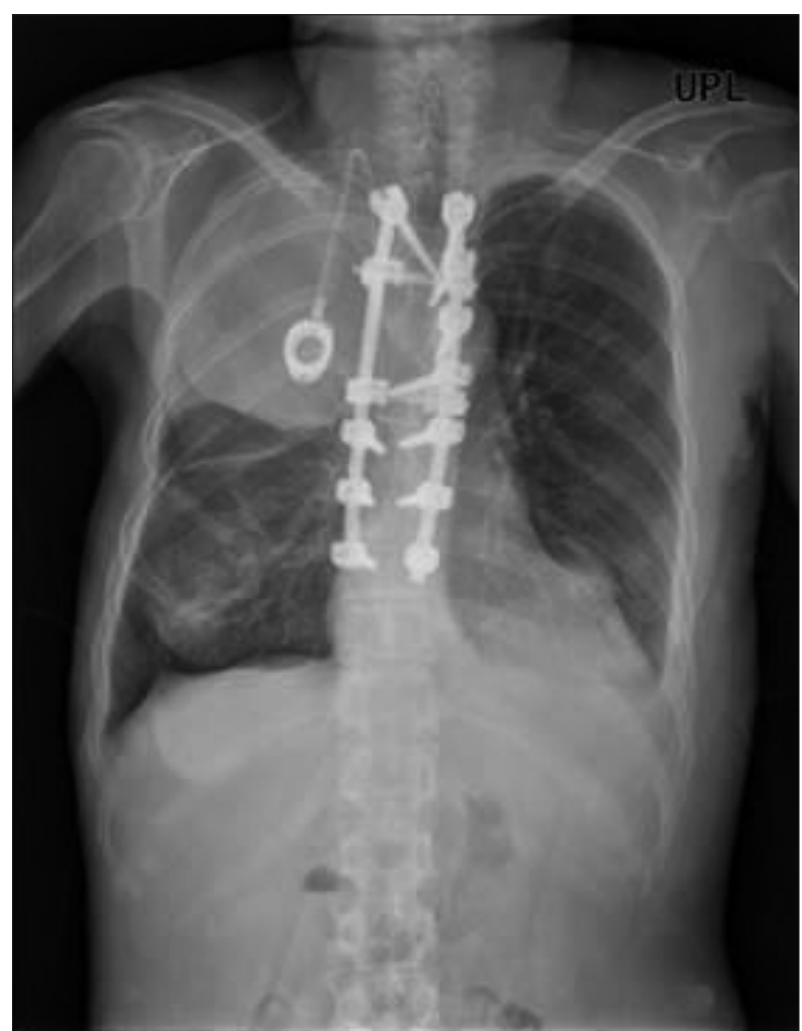

Fig. 6. Follow-up chest $X$-ray showing recurrence of meningocele in the upper lobe of the right lung with satisfactory correction of the kyphoscoliosis.

for meningoceles until they become symptomatic, provided the patients have benign pathology or are surgically difficult cases. Alternatively, some authors ${ }^{1,9)}$ have recommended early surgical intervention for everyone because they observed progressive enlargement of the intrathoracic meningoceles in two-thirds of the cases. However, surgical intervention for intrathoracic meningoceles associated with NF1 is not free from complications including respiratory failure, post-operative CSF leakage, empyema, infection, and recurrence. We were aware of these possible complications preoperatively but chose to perform surgical excision of the meningocele and correction of the spinal deformity due to the presence of acute respiratory distress and neurological deficits. Intervention halted progression of the scoliotic curve and relieved the dyspnea.

Interestingly, spinal deformity is commonly noted in NF-1. Scoliosis is prevalent in approximately $10 \%$ of NF-1 patients, with involvement most commonly observed in the lower cervical and upper thoracic spine ${ }^{16)}$. Scoliosis can be either idiopathic or dystrophic. Additionally, rib penciling, vertebral scalloping, severe apical wedging and rotation, and enlargement of foraminal and paravertebral tumors are frequently observed in neurofibromatosis $^{14)}$. Although patients with NF-1 develop neurological deficits due to spinal dislocation, no optimal surgical procedure for severe scoliosis has been established ${ }^{12)}$. The treatment option for kyphoscoliosis includes two main approaches, posterior-only and a combined anterior and posterior approach.
When the scoliotic angle is between 20 and 40 degrees with normal sagittal curvature (kyphosis $<50$ degrees), also called Type I curve, posterior fusion alone is adequate. However, when the scoliotic angle is greater than 50 degrees with a short, angulated dystrophic curve (Type II curve), more aggressive anterior and posterior spinal fusion is required ${ }^{14)}$. The posterior-only approach involves resection of the bony structures including laminas, spinous processes, transverse processes, and ribs, followed by instrumentation to aid stability. When the posterior approach is attempted, extensive spinal fixation is generally required to prevent progression of post-operative kyphoscolisosis ${ }^{11)}$. In our case, the patient had a large intrathoracic meningocele with a scoliotic angle of approximately 42 degrees with evident dystrophic curvature. The meningocele could have been removed via a transthoracic approach, as the mass itself was large but the transthoracic approach warrants a wide surgical view for total removal of the mass and dural repair ${ }^{5,17)}$. However, probable complications, such as a longer operating time and postoperative pulmonary complications, were considered in depth before attempting transthoracic excision of the mass. Thus, we performed a posterior-only approach for the excision of the meningocele. The spinal deformity was corrected, and fixation was achieved with screws and rods using a derotational technique. About 30 degrees of scoliotic angle was corrected. The operative dead space created after the excision of the mass was sealed with a fat graft to prevent formation of a pseudomeningocele.

Presence of gynecological malignancies in association with NF-1 has been studied by several authors ${ }^{3,13)}$. Among gynecological malignancies, ovarian cancer and breast cancer are most likely to be concomitantly present in patients with NF-1. Since breast cancer is highly associated with mutation of the BRCA1 gene on the long arm of chromosome 17, where the NF-1 gene is also located, co-existence of breast cancer and NF-1 is not unusual. Unfortunately, due to the presence of multiple subcutaneous nodules or skin tumors in NF1 patients, which hinder the detection of breast lumps, early diagnosis of breast cancer can be delayed. Campos et al. ${ }^{2}$ identified that a frame shift mutation of BRCA1 and a nonsense mutation of NF-1 genes results in a four-fold increase in the coexistence of breast cancer and NF-1. In the present case, the patient had been previously diagnosed with NF-1 and was later found to have invasive ductal breast cancer. Early evaluations and treatments are recommended for NF-1 patients when suspicion for breast cancers is high.

\section{CONCLUSION}

The association of intrathoracic meningocele in a patient with NF1 is a rare pathology. Removal of the intrathoracic mass is essential to relieve symptoms of respiratory distress in such situations. However, due to dystrophic curvature already present in these patients, proper spinal fixation procedures are required, implementing either the posterior-only or combined anterior and posterior approach. In our case, we were able to obtain good 
results with a posterior-only approach, and the intrathoracic meningocele was excised successfully. Also, since the gene mutations in both NF-1 and breast cancer occur on the long arm of chromosome 17, concomitance of breast cancer in patients with NF-1 is possible. Hence, proper systemic evaluation and screening, especially of female patients, is warranted to rule out breast cancer while treating NF-1.

\section{References}

1. Akovbiantz A, Hüni R : [Intrathoracic meningocele]. Schweiz Med Wochenschr $101: 19-23,1971$

2. Campos B, Balmaña J, Gardenyes J, Valenzuela I, Abad O, Fàbregas P, et al. : Germline mutations in NF1 and BRCA1 in a family with neurofibromatosis type 1 and early-onset breast cancer. Breast Cancer Res Treat 139: 597-602, 2013

3. Ceccaroni M, Genuardi M, Legge F, Lucci-Cordisco E, Carrara S, D'Amico F, et al. : BRCA1-related malignancies in a family presenting with von Recklinghausen's disease. Gynecol Oncol 86 : 375-378, 2002

4. Chee CP : Lateral thoracic meningocele associated with neurofibromatosis : total excision by posterolateral extradural approach. A case report. Spine (Phila Pa 1976) 14 : 129-131, 1989

5. de Andrade GC, Braga OP, Hisatugo MK, de Paiva Neto MA, Succi E, Braga FM : Giant intrathoracic meningoceles associated with cutaneous neurofibromatosis type I : case report. Arq Neuropsiquiatr 61 (3A) : 677-681, 2003

6. Friedman JM : Epidemiology of neurofibromatosis type 1. Am J Med Genet 89 : 1-6, 1999

7. Laws JW, Pallis C : Spinal deformities in neurofibromatosis. J Bone Joint
Surg Br 45 : 674-682, 1963

8. Miles J, Pennybacker J, Sheldon P : Intrathoracic meningocele. Its development and association with neurofibromatosis. J Neurol Neurosurg Psychiatry 32 : 99-110, 1969

9. Nakaoka K, Takenaka H, Sawada M, Masaoka A : [Intrathoracic meningocele associated with neurofibromatosis (author's transl)]. Kyobu Geka $80: 873-875,1980$

10. Nanson EM : Thoracic meningocele associated with neurofibromatosis. J Thorac Surg $33: 650-662,1957$

11. Oner AY, Uzun M, Tokgöz N, Tali ET : Isolated true anterior thoracic meningocele. AJNR Am J Neuroradiol 25 : 1828-1830, 2004

12. Rockower S, McKay D, Nason S : Dislocation of the spine in neurofibromatosis. A report of two cases. J Bone Joint Surg Am 64 : 1240-1242, 1982

13. Salud A, Porcel JM, Capdevila F, Felip E, Rovirosa MA, del Campo JM : [Ovarian cancer in a female patient with von Recklinghausen's disease]. Med Clin (Barc) $96: 138-140,1991$

14. Singh K, Samartzis D, An HS : Neurofibromatosis type I with severe dystrophic kyphoscoliosis and its operative management via a simultaneous anterior-posterior approach : a case report and review of the literature. Spine J 5 : 461-466, 2005

15. Tonsgard JH : Clinical manifestations and management of neurofibromatosis type 1. Semin Pediatr Neurol 13 : 2-7, 2006

16. Yusuf AS, Pillai A, Menon SK, Panikar D : Massive spontaneous hemothorax, giant intrathoracic meningocele, and kyphoscoliosis in neurofibromatosis type 1. J Surg Tech Case Rep 6 : 33-36, 2014

17. Zamponi C, Cervoni L, Caruso R : Large intrathoracic meningocele in a patient with neurofibromatosis : technical report. Neurosurg Rev 19 : 275-277, 1996 